\title{
Dark matter at the SHiP experiment
}

\author{
Inar Timiryasov ${ }^{1,2, \star}$ \\ ${ }^{1}$ Institute for Nuclear Research of the Russian Academy of Sciences, 60th October Anniversary Prospect, \\ 7a, 117312 Moscow, Russia \\ ${ }^{2}$ Department of Particle Physics and Cosmology, Physics Faculty, Moscow State University, Vorobievy Gory, \\ 119991 Moscow, Russia
}

\begin{abstract}
We study prospects of dark matter searches in the SHiP experiment. SHiP (Search for Hidden Particles) is the recently proposed fixed target experiment which will exploit the high-intensity beam of $400 \mathrm{GeV}$ protons from the CERN SPS. In addition to the hidden sector detector, SHiP will be equipped with the $v_{\tau}$ detector, which presumably would be sensitive to dark matter particles. We describe appropriate production and detection channels and estimate SHiP's sensitivity for a scalar dark matter coupled to the Standard model through the vector mediator.
\end{abstract}

\section{Introduction}

We address the question of a possibility of dark matter (DM) search in the SHiP experiment. SHiP (Search for Hidden Particles) is the recently proposed [1,2] fixed target experiment exploiting 400 $\mathrm{GeV}$ proton beam from the CERN SPS. The original motivation of the experiment [3] was the search for $O(1) \mathrm{GeV}$ sterile neutrinos of the $v \mathrm{MSM}$ (see [4] for review of the model). Singlet fermions and active neutrinos are mixed and this mixing is responsible for both production of singlets in decays of heavy mesons (generated by protons on target) and subsequent singlet decays into SM particles (the main signature for the SHiP detector), see [5] for details. The flux of secondary charged particles (mostly muons) is suppressed by the very dense shielding placed downstream. The main idea is to place a large detector $\left(5 \times 10 \mathrm{~m}^{2} \times 50 \mathrm{~m}[2]\right)$ as close to the target as possible (at a distance of about $60 \mathrm{~m} \mathrm{[2])} \mathrm{in} \mathrm{order} \mathrm{to} \mathrm{maximize} \mathrm{covered} \mathrm{solid} \mathrm{angle.} \mathrm{This} \mathrm{setup} \mathrm{makes} \mathrm{SHiP} \mathrm{be} \mathrm{a} \mathrm{universal} \mathrm{tool} \mathrm{to}$ probe any models of new physics containing light and long-lived particles which could be produced by protons on target and then decaying into SM particles [1, 6, 7].

The SHiP experiment will be equipped with a tau neutrino detector. In addition to it's main purpose - the first direct observation of $\bar{v}_{\tau}$ - the tau neutrino detector will be capable of observing light DM particles, produced in the beam dump. The possibility of this type searches was briefly discussed in Refs. [1, 2]. In what follows we quote relevant production rates and cross sections and estimate expected sensitivity of the SHiP.

^e-mail: timiryasov@inr.ac.ru 


\section{Dark matter candidate}

Models of light dark matter (DM) usually contain light mediator particles. Such mediators are required to provide the realistic value of DM abundance in the Universe [8]. The presence of these mediators provides a possibility to produce a beam of DM particles at fixed target experiments. These DM particles, in turn, could be detected in neutrino detectors. SHiP setup contains all elements required to provide that type of DM search: a high-intensity proton beam, a target designed to suppress neutrino background and $v_{\tau}$ detector consisting of OPERA-type bricks employing Emulsion Cloud Chamber (ECC) technology (see Ref. [2] for details). DM candidate assumed to be a complex scalar $\chi$. These particles interact with SM particles through the exchange of vector boson $A^{\prime}$. We assume that $m_{A^{\prime}}>$ $2 m_{\chi}$, so that decay $A^{\prime} \rightarrow \chi \chi \chi^{\dagger}$ is kinematically allowed and contributes to the invisible decay mode of $A^{\prime}$.

The simplest, say, toy Lagrangian of this type with extra $U^{\prime}(1)$ group reads:

$$
\mathcal{L}=\mathcal{L}_{S M}-\frac{1}{4} F_{\mu \nu}^{\prime} F^{\mu \nu}+\frac{\epsilon}{2} F_{\mu \nu}^{\prime} F^{\mu \nu}+\frac{m_{A^{\prime}}^{2}}{2} A_{\mu}^{\prime} A^{\prime \mu}+\left|D_{\mu} \chi\right|^{2}-m_{\chi}^{2}|\chi|^{2}
$$

where $\chi$ and $A^{\prime}$ are DM and mediator fields, $F_{\mu \nu}=\partial_{\mu} A_{v}^{\prime}-\partial_{v} A_{\mu}^{\prime}, D_{\mu}=\partial_{\mu}+i e^{\prime} A_{\mu}^{\prime}, e^{\prime}$ is the $U^{\prime}(1)$ coupling constant and $\epsilon$ is the parameter of kinematic mixing. In the Eq. (1) we have directly written mass term $m_{A^{\prime}}$ for the vector mediator, however, we suppose that this mass term stems from the extra-Higgs mechanism in the dark sector. Note that this model, even in its renormalizable form with explicit extra-Higgs mechanism, is not free from anomalies. However, the model is attractive since the whole phenomenology is controlled only by four parameters: $\epsilon, e^{\prime}, m_{A^{\prime}}, m_{\chi}$.

We consider scalar DM candidate since it exhibits $p$-wave annihilation, which is crucial to satisfying cosmological constraints. At the same time, fermion coupled through a massive vector implies $s$-wave annihilation. Thus it could be only sub-dominant component of a DM.

\section{Estimate of dark matter flux}

In the SHiP setup, DM particles $\chi$ could be produced directly in the proton-proton, or proton-neutron collisions via s-channel exchange of the hidden photon $A^{\prime}$. If the hidden photon is sufficiently longlived and $\Gamma\left(A^{\prime}\right) \ll m_{A^{\prime}}$, then one can use the narrow width approximation. In this approximation $\chi$ production cross section factorizes,

$$
\sigma(p N \rightarrow \chi \bar{\chi})=\sigma\left(p N \rightarrow A^{\prime}\right) \operatorname{Br}\left(A^{\prime} \rightarrow \chi \bar{\chi}\right),
$$

and the flux of DM particles $\Phi_{\chi}=2 \Phi_{A^{\prime}} \operatorname{Br}\left(A^{\prime} \rightarrow \chi \bar{\chi}\right)$. Therefore, in this case the problem of DM flux computation reduces to that of $A^{\prime}$ flux computation.

Despite the popularity of the vector mediator framework, there is no generally accepted method of its production rate computation. The Weizsäcker-Williams approximation was employed in Ref. [9] in order to estimate the flux of $A^{\prime}$ produced in the process of proton bremsstrahlung. This method deals with the entire proton. Therefore, in order to be sure that the method works properly one should introduce the proton form factors [10]. These form factors sharply drop (as $q^{-4}$, where $q$ is 4-momentum transfer) for $A^{\prime}$ heavier than $O(1) \mathrm{GeV}$ and lead to underestimation of the flux of heavy vector mediators.

In order to estimate the flux of hidden photons we employ results of Ref. [10], where two different mechanisms of $A^{\prime}$ production were considered:

1) proton bremsstrahlung 
2) radiative decays of secondary mesons $\left(\pi^{0}\right.$ and $\left.\eta\right)$.

The average energy of the DM particles, produced via the proton bremsstrahlung is

$$
\left\langle E_{\text {in }}\right\rangle \simeq(170-190)\left(\frac{m_{\chi}}{m_{A^{\prime}}}\right) \mathrm{GeV}, \quad \text { for } m_{A^{\prime}} \text { in range } 10^{-4}-5 \mathrm{GeV}
$$

The production cross section (and the flux) is proportional to $\propto \epsilon^{2}$. The number of DM particles produced by $10^{20}$ protons on target via bremsstrahlung is shown in Fig. 1 as function of mediator mass $m_{A^{\prime}}$ for a fixed value of $\epsilon=10^{-4}$.

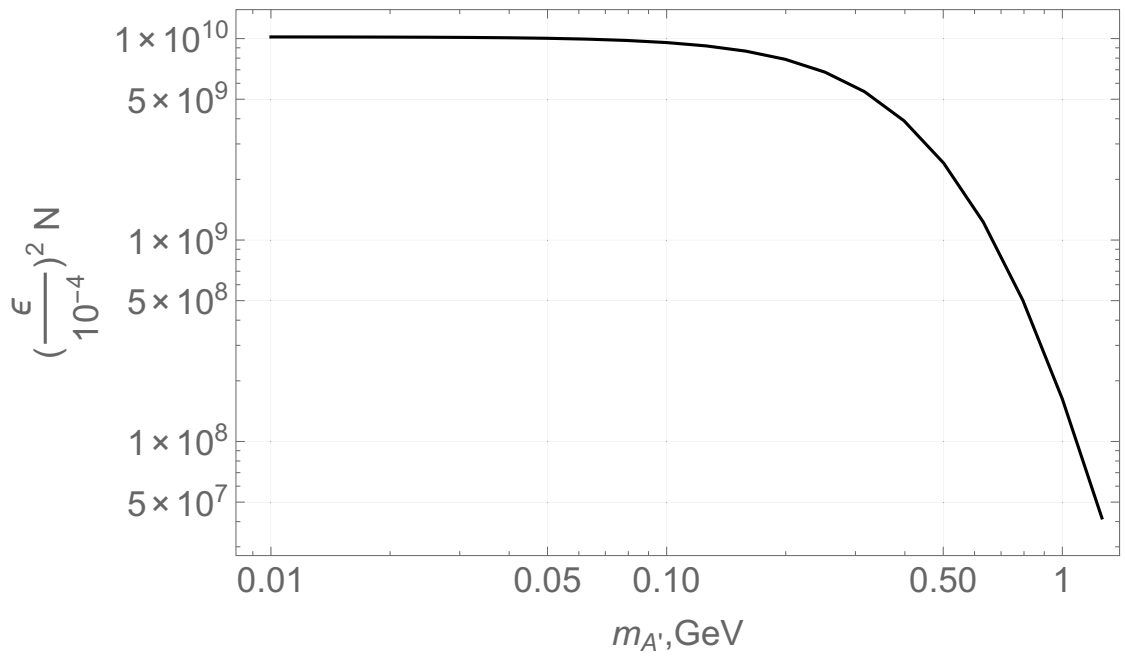

Figure 1. Number of DM particles $N_{\chi}$ which will traverse the detector (i. e. with $\theta<0.025$, where $\theta$ is the angle between the direction of particle's 3-momenta and the beam direction) as function of the mediator mass $m_{A^{\prime}}$ for $\epsilon=10^{-4}$. We assume that $\operatorname{Br}\left(A^{\prime} \rightarrow \chi \bar{\chi}\right) \simeq 1$.

The mixing $\epsilon$ is responsible for $A^{\prime}$ decays into pairs of charged SM particles, while $e^{\prime}$ governs decay into DM scalars $\chi$. The partial decay width into a lepton pair is given by

$$
\Gamma_{A^{\prime}}^{l^{+} l^{-}}=\frac{1}{3} \alpha m_{A^{\prime}} \epsilon^{2} \sqrt{1-\frac{4 m_{l}^{2}}{m_{A^{\prime}}^{2}}}\left(1+\frac{2 m_{l}^{2}}{m_{A^{\prime}}^{2}}\right),
$$

where $m_{l}$ is the mass of the lepton and $\alpha$ is the fine structure constant.

The partial decay width into scalar DM particles is given by

$$
\Gamma_{A^{\prime}}^{\chi \chi^{\dagger}}=\frac{1}{4} \alpha^{\prime} m_{A^{\prime}}\left(1-\frac{4 m_{\chi}^{2}}{m_{A^{\prime}}^{2}}\right)^{3 / 2}
$$

where $\alpha^{\prime} \equiv e^{\prime 2} / 4 \pi$. In what follows assume that $\alpha^{\prime} \gg \alpha \epsilon^{2}$. 


\section{Dark matter scattering cross sections}

Dark matter particles could scatter both on electrons and nucleons via the exchange of light mediator.

The elastic DM-electron scattering cross section has the following form [12]:

$$
\frac{d \sigma_{\chi e \rightarrow \chi e}}{d E_{e}}=4 \pi \epsilon^{2} \alpha \alpha^{\prime} \frac{2 m_{e} E_{i n}^{2}-\left(2 m_{e} E_{i n}+m_{\chi}^{2}\right)\left(E_{e}-m_{e}\right)}{\left(E_{i n}^{2}-m_{\chi}^{2}\right)\left(m_{A^{\prime}}^{2}+2 m_{e} E_{e}-2 m_{e}^{2}\right)^{2}},
$$

where $E_{e}$ is the energy of the recoil electron and $E_{i n}$ is the energy of the incident dark matter particle and $\alpha^{\prime} \equiv e^{\prime 2} / 4 \pi$.

The lowest order scalar DM-nucleon cross section was calculated in Ref. [13] and reads (for proton and neutron $N=p, n$, respectively):

$$
\frac{d \sigma_{\chi N \rightarrow \chi N}}{d Q^{2}}=4 \pi \epsilon^{2} \alpha \alpha^{\prime} \frac{F^{2}\left(Q^{2}\right)\left[q_{N}^{2} A\left(E_{i n}, Q^{2}\right)-\frac{1}{4} \kappa_{N}^{2} B\left(E_{i n}, Q^{2}\right)\right]}{2 m_{N}\left(m_{A^{\prime}}+Q^{2}\right)^{2}\left(E_{\text {in }}^{2}-m_{\chi}^{2}\right)},
$$

where $Q^{2}=2 m_{N}\left(E_{\text {in }}-E_{\chi}\right)$ is the momentum transfer, $E_{\chi}$ is the energy of the outgoing DM particle, form factor in the simplest form is $F=1 /\left(1+Q^{2} / m_{N}^{2}\right)^{2}$ with $q_{p}=1, q_{n}=0, \kappa_{p}=1.79$ and $\kappa_{n}=-1.9$. The functions $A$ and $B$ are defined as:

$$
\begin{array}{r}
A\left(E_{i n}, Q^{2}\right)=2 m_{N} E_{i n}\left(E_{i n}-Q^{2} / 2 m_{N}\right)-m_{\chi}^{2} Q^{2} / 2 m_{N}, \\
B\left(E_{i n}, Q^{2}\right)=\left(Q^{2} / 2 m_{N}\right)\left[\left(2 E_{\text {in }}-Q^{2} / 2 m_{N}\right)^{2}+Q^{2}-4 m_{\chi}^{2}\right] .
\end{array}
$$

It is known (see, e.g. Fig 4.1 in Ref. [14]) that the neutrino-proton cross section is approximately two orders magnitude greater than the neutrino-electron cross section for neutrino energies greater than hundreds $\mathrm{MeV}$. In order to determine the most relevant interaction pattern for the SHiP setup, we present the total cross sections of two processes described above in Fig. 2. To obtain these total cross sections we integrate the differential cross sections (6) and (7) over the allowed kinematical range (described in Appendix A) and apply an additional cut on the recoil energy of the target particle $E_{T}>E_{\text {cut }}=1 \mathrm{GeV}$.

As one can see from Fig. 2, $\chi-p$ total cross section is more than one order magnitude greater than that of $\chi-e$ scattering. However, one can see from Fig. 3, that the $\chi-p$ differential cross section is steeply falls with the $Q^{2}$. Therefore the resulting signal rate of the $\chi$-nucleon scattering will depend on the assumed cut on the nucleons recoil energy $E_{\text {cut }}$.

\section{Signal calculation}

For the reason described above, we will consider only $\chi-e$ scattering and assume that the energy of the recoil electron $E_{e}>E_{\text {cut }}=1 \mathrm{GeV}$.

We consider production of DM particles $\chi$ in decays of hidden photons $A^{\prime}: A^{\prime} \rightarrow \chi \chi^{\dagger}$. Hidden photons, in turn, could be produced via the proton bremsstrahlung or in decays of neutral mesons. These production channels were studied in Ref. [10] and we employ the flux of $A^{\prime}$ calculated in this work. We compute energy-angle distribution of $\chi$ for $m_{\chi}=0.1 \mathrm{MeV}$ and for $m_{A^{\prime}}$ in range $\left(10^{-4}-1\right)$ $\mathrm{GeV}$. Using this distribution we determine the signal rate:

$$
N_{s i g}=\int_{d e t} d \Omega \int d E_{\text {in }} n \sigma\left(E_{\text {in }}\right) L_{d e t} \frac{d N_{\chi}}{d E_{\text {in }} d \Omega},
$$

where number of electrons per volume $n=Z N_{A} \rho / A$, and $Z$ is atomic number, $N_{A}$ is Avogadro's number, $\rho$ is density of the medium and $A$ is mass of a mole. We assume that the length of the lead 


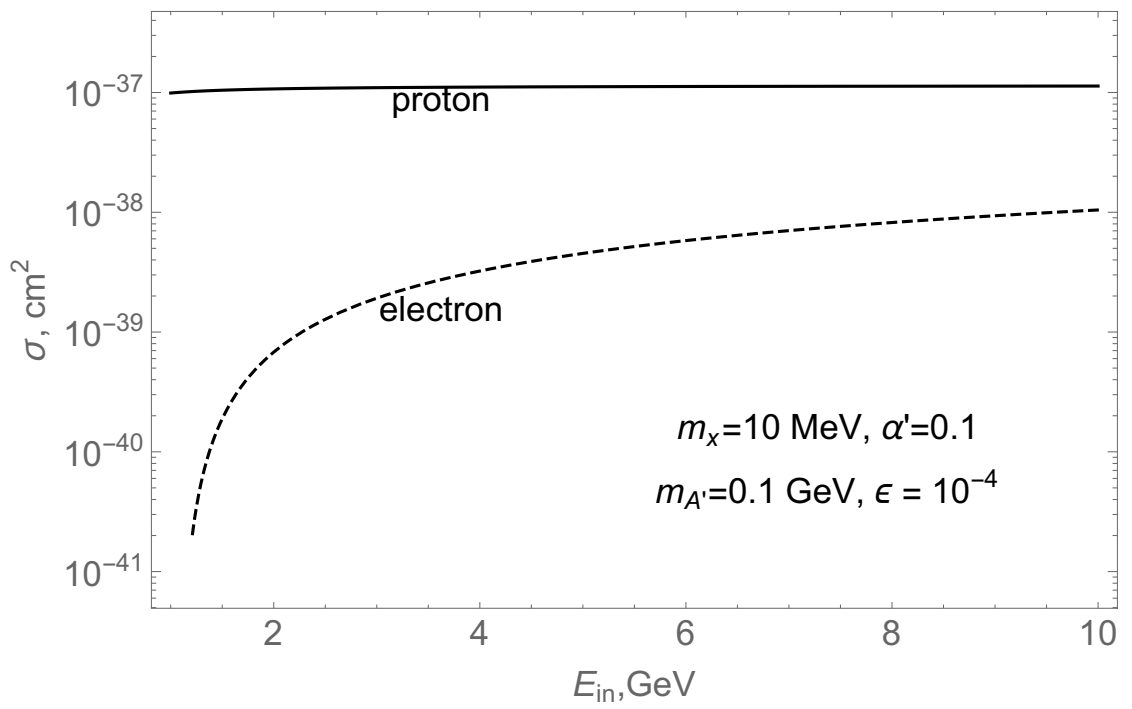

Figure 2. $\sigma_{\chi e \rightarrow \chi e}$ and $\sigma_{\chi N \rightarrow \chi N}$ as function of the incident $\chi$ energy. We have applied the cut on the final energy of the target (electron or proton): $E_{T}>1 \mathrm{GeV}$.

detector is $L_{d e t}=100 \mathrm{~cm}$. The result for $N_{\text {sig }}=10$ and $10^{20}$ protons on target is shown in Fig. 4 . The dark gray region corresponds to $A^{\prime}$ produced via the proton bremsstrahlung while light gray region corresponds to $A^{\prime}$ produced in decays of secondary mesons.
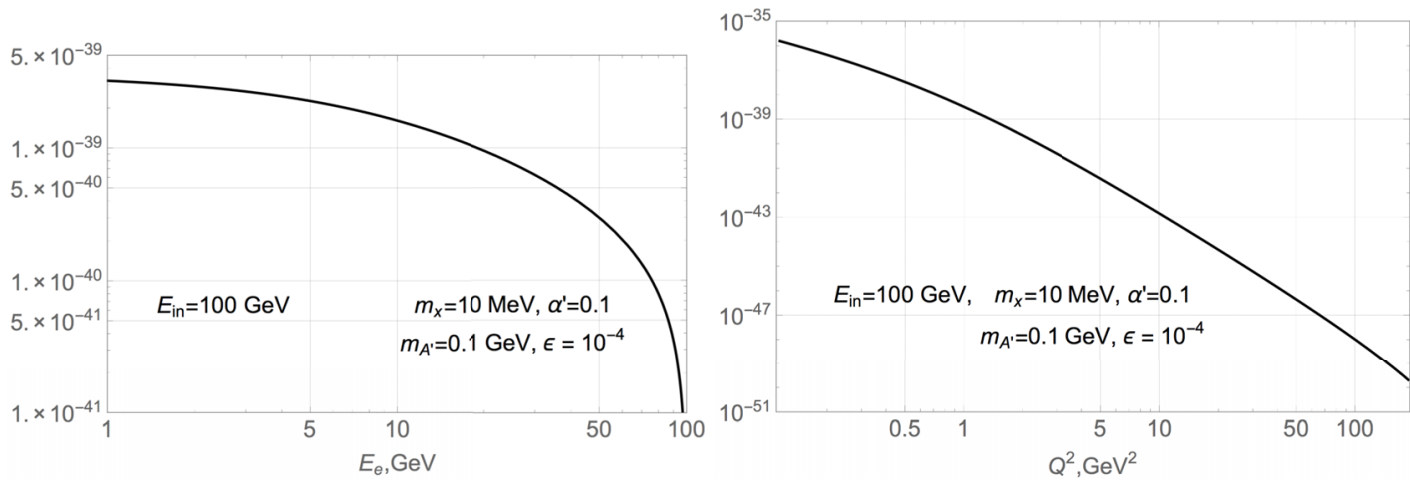

Figure 3. Left panel: differential cross section of $\chi$ - electron scattering (6) as function of recoil electron energy $E_{e}$. Right panel: differential cross section of $\chi$ - proton scattering (7) as function of the momentum transfer $Q^{2}$. The energy of incident DM particle $E_{i n}=100 \mathrm{GeV}$. We have applied a cut on the final energy of the target (electron or proton): $E_{T}>1 \mathrm{GeV}$. Reference values of parameters, which were used in this plots, are not excluded by present experiments. 


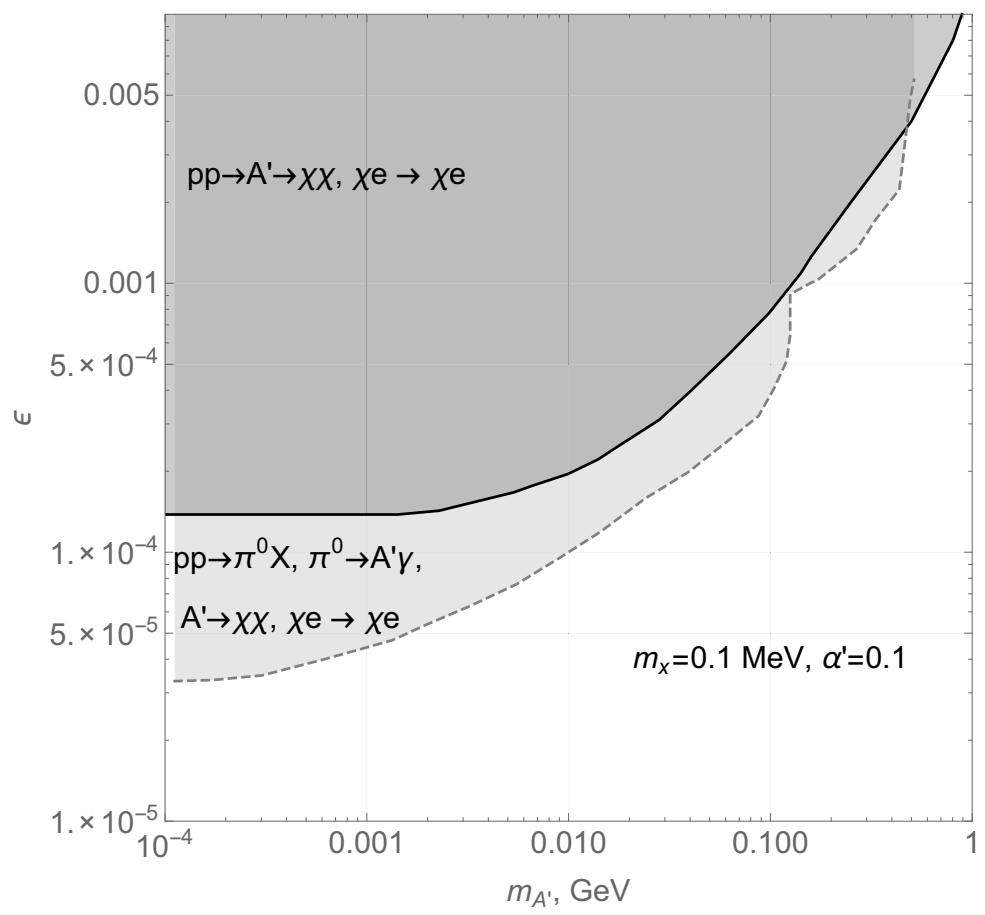

Figure 4. Estimate of SHiP's sensitivity to scalar DM $\chi$ (10 signal events, $10^{20}$ protons on target) in $m_{A^{\prime}}-\epsilon$ plane. The dark gray region corresponds to $A^{\prime}$ produced via the proton bremsstrahlung while light gray region corresponds to $A^{\prime}$ produced in decays of secondary mesons.

\section{Conclusions}

To summarize, we have estimated the flux of light dark matter particles expected in the SHiP experiment and outlined the region in the model parameter space (see Fig. 4), where $10 \chi$-e scatterings with $E_{e}>1 \mathrm{GeV}$ are expected for each $10^{20}$ protons on target. We assert that the SHiP experiment provides a possibility to search for light dark matter particles. The high energy of incident protons together with a huge statistics allows investigating yet not reached regions of the parameter space. A promising channel of dark matter - proton scattering deserves further studies. One needs to estimate, what is the minimal value of transferred momentum $Q_{\min }^{2}$ required to separate the signal from the background induced by elastic neutrino scatterings.

\section{Acknowledgments}

The author is indebted to D. S. Gorbunov for useful discussions and inspiration. The author is grateful to N. G. Polukhina and N. I. Starkov for valuable comments and interest to the work. This work has been supported by Russian Science Foundation grant 14-22-00161. 


\section{Appendix A: Kinematics}

In order to calculate a total cross section of DM scattering one need to know an appropriate integration limits. In this Appendix we present some relevant details.

Consider the process $\chi\left(p_{a}\right)+T\left(p_{b}\right) \rightarrow \chi\left(p_{1}\right)+T\left(p_{2}\right)$, where $T$ is a target particle of mass $m_{T}$, $T=e, p, n$. Standard Mandelstam variables reads:

$$
\begin{aligned}
& s=\left(p_{a}+p_{b}\right)^{2}=\left(p_{1}+p_{2}\right)^{2}=m_{\chi}^{2}+m_{T}^{2}+2 m_{T} E_{a}, \\
& t=\left(p_{a}-p_{1}\right)^{2}=\left(p_{b}-p_{2}\right)^{2}=2 m_{T}^{2}-2 m_{T} E_{2}, \\
& u=\left(p_{a}-p_{2}\right)^{2}=\left(p_{b}-p_{1}\right)^{2}=m_{\chi}^{2}+m_{T}^{2}-2 m_{T} E_{1} .
\end{aligned}
$$

From (9) and $s+t+u=2 m_{\chi}^{2}+2 m_{T}^{2}$ one immediately obtains two useful forms for the momentum transfer:

$$
\begin{aligned}
& Q^{2}=-t=2 m_{T}\left(E_{a}-E_{1}\right), \\
& Q^{2}=-t=2 m_{T}\left(E_{2}-m_{T}\right) .
\end{aligned}
$$

The integration limits in terms of $E_{1}$ or $E_{2}$ are defined by the physical region of $2 \rightarrow 2$ scattering:

$$
-\frac{\lambda\left(s, m_{\chi}^{2}, m_{T}^{2}\right)}{s} \leq t \leq 0
$$

where $\lambda(x, y, z)=(x-y-z)^{2}-4 y z$ is ordinary Källén triangle function.

Requiring $E_{2}>E_{\text {cut }}$ one also gets $t \leq-2 m_{T}\left(E_{\text {cut }}-m_{T}\right)$.

Finally,

$$
\begin{aligned}
E_{a}-\frac{2 m_{T}}{s}\left(E_{a}^{2}-m_{T}^{2}\right) & \leq E_{1} \leq E_{a}+m_{T}-E_{c u t} \\
E_{c u t} & \leq E_{2} \leq m_{T}+\frac{2 m_{T}}{s}\left(E_{a}^{2}-m_{T}^{2}\right)
\end{aligned}
$$

\section{References}

[1] S. Alekhin et al., arXiv:1504.04855 [hep-ph].

[2] M. Anelli et al. [SHiP Collaboration], arXiv:1504.04956 [physics.ins-det].

[3] S. N. Gninenko, D. S. Gorbunov and M. E. Shaposhnikov, Adv. High Energy Phys. 2012 (2012) 718259 doi:10.1155/2012/718259 [arXiv:1301.5516 [hep-ph]].

[4] A. Boyarsky, O. Ruchayskiy and M. Shaposhnikov, Ann. Rev. Nucl. Part. Sci. 59 (2009) 191 doi:10.1146/annurev.nucl.010909.083654 [arXiv:0901.0011 [hep-ph]].

[5] D. Gorbunov and M. Shaposhnikov, JHEP 0710 (2007) 015 Erratum: [JHEP 1311 (2013) 101] doi:10.1007/JHEP11(2013)101, 10.1088/1126-6708/2007/10/015 [arXiv:0705.1729 [hep-ph]].

[6] D. Gorbunov and I. Timiryasov, Phys. Rev. D 92 (2015) no.7, 075015 doi:10.1103/PhysRevD.92.075015 [arXiv:1508.01780 [hep-ph]].

[7] K. O. Astapov and D. S. Gorbunov, Phys. Rev. D 93 (2016) no.3, 035008 doi:10.1103/PhysRevD.93.035008 [arXiv:1511.05403 [hep-ph]].

[8] M. Pospelov, A. Ritz and M. B. Voloshin, Phys. Lett. B 662 (2008) 53 doi:10.1016/j.physletb.2008.02.052 [arXiv:0711.4866 [hep-ph]].

[9] J. Blümlein and J. Brunner, Phys. Lett. B 731 (2014) 320 doi:10.1016/j.physletb.2014.02.029 [arXiv:1311.3870 [hep-ph]]. 
[10] D. Gorbunov, A. Makarov and I. Timiryasov, Phys. Rev. D 91 (2015) no.3, 035027 doi:10.1103/PhysRevD.91.035027 [arXiv:1411.4007 [hep-ph]].

[11] K. A. Olive et al. [Particle Data Group Collaboration], Chin. Phys. C 38 (2014) 090001. doi:10.1088/1674-1137/38/9/090001

[12] B. Batell, R. Essig and Z. Surujon, Phys. Rev. Lett. 113 (2014) no.17, 171802 doi:10.1103/PhysRevLett.113.171802 [arXiv:1406.2698 [hep-ph]].

[13] P. deNiverville, D. McKeen and A. Ritz, Phys. Rev. D 86 (2012) 035022 doi:10.1103/PhysRevD.86.035022 [arXiv:1205.3499 [hep-ph]].

[14] A. Strumia and F. Vissani, hep-ph/0606054. 